\title{
On the power values of Stirling numbers
}

\author{
by \\ B. Brindza* ANd Á. Pintér (Debrecen)
}

1. Introduction. The arithmetical properties of certain combinatorial numbers and diophantine equations involving binomial coefficients have been investigated by several authors. The purpose of the present paper is to establish some effective finiteness results on the power values of Stirling numbers of first and second kind. The proofs are mainly based on the theory of linear forms in logarithms. We combine these profound results with some elementary tricks on the factorization of certain polynomials and with well-known theorems on superelliptic and unit equations.

Following the usual notation, let $S_{k}^{n}$ denote the Stirling number of second kind with parameters $(n, k)$, that is, $S_{k}^{n}$ is the number of partitions of the set $\{1,2, \ldots, n\}$ into $k$ non-empty subsets. Moreover, we denote by $s_{k}^{n}$ the Stirling number of first kind with parameters $(n, k)$, that is, $s_{k}^{n}$ is the number of permutations of $n$ elements which are products of $k$ disjoint cycles.

Then we have the following extension of a result of Pintér [4].

TheOrem 1. Let $a$ and $b$ be positive integers. Then all solutions of both the equations

$$
S_{x-a}^{x}=b y^{z} \quad \text { and } \quad s_{x-a}^{x}=b y^{z},
$$

in rational integers $x, y, z$ with $x>2 b 16^{a} a^{8 a},|y|>1, z \geq 2$, satisfy

$$
\frac{z}{(7.5+\log z)^{2}}<11000(\log b+8 a \log a+3 a) .
$$

Furthermore, (i) if $z \geq 3$ or (ii) if $z=2$ and $a \notin\{1,3\}$, then $\max \{x,|y|\}<$ $c_{1}$, where $c_{1}$ is an effective constant depending only on $a$ and $b$.

Remarks. If the technical condition on the magnitude of $x$ does not hold then we have reasonable bounds for $x$ and $y$, too, without large constants. Namely, $x \leq 2 b 16^{a} a^{8 a}$ and relations (a), (a'), (4) and (5) (see Sec-

* Research supported in part by Grant 273 from the Hungarian National Foundation for Scientific Research. 
tions 2 and 3) imply

$$
y^{z} \leq b^{2 a-1} a^{16 a^{2}+2 a} 2^{8 a^{2}+3 a} .
$$

The restriction made on $a$ is necessary in the case (ii) since the equations

$$
\left(\begin{array}{l}
x \\
2
\end{array}\right)=S_{x-1}^{x}=y^{2}, \quad \frac{1}{2}\left(\begin{array}{l}
x \\
4
\end{array}\right)(x-2)(x-3)=S_{x-3}^{x}=y^{2}
$$

and

$$
\left(\begin{array}{l}
x \\
2
\end{array}\right)=s_{x-1}^{x}=y^{2}, \quad\left(\begin{array}{l}
x \\
4
\end{array}\right)\left(\begin{array}{l}
x \\
2
\end{array}\right)=s_{x-3}^{x}=y^{2}
$$

have infinitely many solutions.

TheOREM 2. All the solutions of the equation

$$
S_{a}^{x}=b y^{z} \quad \text { with } \quad a>1, x>a,|y|>1
$$

satisfy $z<c_{2}$ where $c_{2}$ is an effective constant depending only on $a$ and $b$.

After the auxiliary results it will be clear that this equation is a harder problem than equation (1).

2. Preliminaries. To prove the above theorems we need some general results from the theory of diophantine equations, moreover, several properties of Stirling numbers.

Let $\mathbf{K}$ be a finite extension of $\mathbf{Q}$ with ring of integers $\mathcal{O}_{\mathbb{K}}$. Suppose that $a_{0}, a_{1}, \ldots, a_{d}(d \geq 1)$ are distinct algebraic numbers in $\mathbf{K}$. Write

$$
f(x)=a_{0}\left(x-a_{1}\right)^{r_{1}} \ldots\left(x-a_{d}\right)^{r_{d}}
$$

where $r_{1}, \ldots, r_{d}$ are positive rational integers. Further, let $m \geq 2$ be a natural number and put $t_{i}=m /\left(m, r_{i}\right)$ for $1 \leq i \leq d$.

We consider the superelliptic equation

$$
f(x)=y^{m} \quad \text { in } x, y \in \mathcal{O}_{\mathbb{K}} .
$$

The next lemma is a special case of a result of Brindza.

Lemma 1 (Brindza [1], Theorem). Suppose that $\left\{t_{1}, \ldots, t_{d}\right\}$ is not a permutation of either of the d-tuples $(t, 1, \ldots, 1), t \geq 1$, and $(2,2,1, \ldots, 1)$. Then all solutions $(x, y) \in \mathcal{O}_{\mathbb{K}}^{2}$ of the equation (3) satisfy $\left({ }^{1}\right)$

$$
\max \{H(x), H(y)\}<c_{3}
$$

where $c_{3}$ is an effectively computable constant depending only on $\mathbf{K}, f(x)$ and $m$.

$\left({ }^{1}\right)$ By the height $H(\alpha)$ of an algebraic number $\alpha$ we mean, as usual, the maximum of the absolute values of the relatively prime integer coefficients in its minimal defining polynomial. 
The following theorem is a simple special case of a result of Mignotte and Waldschmidt and will play the main rôle in the proof of Theorem 1.

Lemma 2 (Mignotte and Waldschmidt [3], Corollary 1.1 and subsequent remark). Let $\alpha_{1}, \alpha_{2}$ be positive rational numbers and let $b_{1}, b_{2}$ be positive rational integers such that

$$
\alpha_{1}^{b_{1}} \neq \alpha_{2}^{b_{2}}
$$

Then

$$
\left|\alpha_{1}^{b_{1}} \alpha_{2}^{-b_{2}}-1\right|>\exp \left\{-500 H_{1} H_{2}(7.5+\log B)^{2}\right\}
$$

where $B=\max \left(b_{1}, b_{2}\right)$ and $H_{i}=\max \left\{\log 2 H\left(\alpha_{i}\right), 2 e\left|\log \alpha_{i}\right|, 1\right\}$ for $i=1$ and 2 .

Let $p \geq 3, w_{1}, \ldots, w_{p}$ be non-zero distinct rational integers, and let $q_{1}, \ldots, q_{p}$ be non-zero rational numbers and put

$$
u_{n}=q_{1} w_{1}^{n}+\ldots+q_{p} w_{p}^{n} \quad \text { for } n \geq 0 .
$$

The next lemma is a simple consequence of some related results of Shorey and Stewart ([8], [9]).

Lemma 3. If $\left|w_{1}\right|>\left|w_{2}\right|>\left|w_{j}\right|$ for $j=3, \ldots, p$ and $u_{n}=b y^{z}$ for integers $b$ and $y$ and $z$ larger than one, then $z$ is less than $c_{4}$, a number which is effectively computable in terms of $b, q_{1}, \ldots, q_{p}$ and $w_{1}, \ldots, w_{p}$.

Proof. See Shorey and Stewart [8], Theorem 3, and [9], Corollary 1.

Let $\widetilde{S}_{k}^{n}$ denote the number of partitions of $\{1,2, \ldots, n\}$ into $k$ subsets such that every subset contains at least two elements. For the relations (a), (b), (d) below we refer, for instance, to Problems 189, 203 and 204 in Part 1 of Pólya-Szegö [5], and equation (c) immediately comes from the definitions and (b). At this stage we remark that, recently, Howard [2] has obtained some new results on divisibility properties of Stirling numbers.

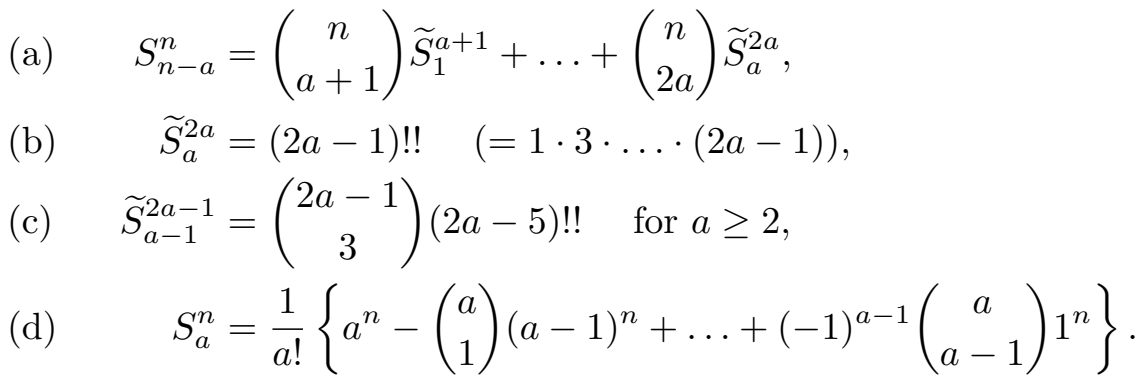

Similar relations hold for Stirling numbers of first kind, namely,

$\left(\mathrm{a}^{\prime}\right) s_{n-a}^{n}=\left(\begin{array}{c}n \\ a+1\end{array}\right) \widetilde{s}_{1}^{a+1}+\ldots+\left(\begin{array}{c}n \\ 2 a\end{array}\right) \widetilde{s}_{a}^{2 a} \quad$ (see e.g. [6], pp. $\left.72-74\right)$, 
where the so-called associated Stirling numbers of first kind satisfy the relations

$$
\widetilde{s}_{k}^{n}=(n-1) \cdot \widetilde{s}_{k}^{n-1}+(n-1) \cdot \widetilde{s}_{k-1}^{n-2},
$$

with

$$
\begin{array}{ll}
\widetilde{s}_{0}^{0}=1, & \\
\widetilde{s}_{0}^{n}=0 & \text { if } n>0, \\
\widetilde{s}_{1}^{n}=(n-1) ! & \text { if } n>1, \\
\widetilde{s}_{k}^{n}=0 & \text { if } n<2 k \text { or } k<0 .
\end{array}
$$

One can see by induction that

$$
\begin{aligned}
\widetilde{s}_{a}^{2 a} & =(2 a-1) ! !, \\
\widetilde{s}_{a-1}^{2 a-1} & =2\left(\begin{array}{c}
2 a-1 \\
3
\end{array}\right)(2 a-5) ! ! \quad \text { for } a \geq 2 .
\end{aligned}
$$

From (b), (c) and $\left(b^{\prime}\right),\left(c^{\prime}\right)$ we obtain

(e) $\frac{\widetilde{S}_{a-1}^{2 a-1}}{\widetilde{S}_{a}^{2 a}}<\frac{(a-1)^{2}}{2 a}$ and $\quad\left(\mathrm{e}^{\prime}\right) \quad \frac{\widetilde{s}_{a-1}^{2 a-1}}{\widetilde{s}_{a}^{2 a}}>\frac{a^{2}-1}{2 a}$

for $a>3$.

LEMma 4. Let $b_{1}, \ldots, b_{a}$ be non-zero real numbers. If $a>1$ and

then the polynomial

$$
\frac{b_{a-1}}{b_{a}} \notin\left[\frac{(a-1)^{2}}{2 a}, \frac{a^{2}-1}{2 a}\right]
$$

$$
P(x)=b_{1}\left(\begin{array}{c}
x \\
a+1
\end{array}\right)+\ldots+b_{a-1}\left(\begin{array}{c}
x \\
2 a-1
\end{array}\right)+b_{a}\left(\begin{array}{c}
x \\
2 a
\end{array}\right)
$$

has at least three zeros with odd multiplicity.

It is known that every non-constant polynomial can be expressed as a linear combination of the polynomials $p_{n}(x)=x(x-1) \ldots(x-n), n=$ $0,1,2, \ldots$ Hence it is a little bit surprising that our condition on $b_{a-1} / b_{a}$ is enough to guarantee the existence of three zeros with odd multiplicity.

Proof. We can write $P(x)$ in the form $P(x)=\left(\begin{array}{c}x \\ a+1\end{array}\right) Q(x)$ where the degree of $Q(x)$ is $a-1$. Hence $P(x)$ has at least two simple zeros. Moreover, we may assume that every zero of $Q(x)$ belongs to the set $\{0,1, \ldots, a\}$, for otherwise $P(x)$ has at least three simple zeros. If $P(x)$ has a zero with multiplicity at least four then $P(x)$ has three simple zeros again. Indeed, supposing the contrary we have $\operatorname{deg} P \geq 2 \cdot 1+4+2(a-2)>2 a$. If $P(x)$ has a zero with multiplicity three then Lemma 4 is proved, hence the only remaining case is when $P(x)$ has two simple zeros and all the other zeros 
have multiplicity two. Then we obtain

$$
P(x)=\frac{b_{a}}{(2 a) !}(x-i)(x-j) \prod_{\substack{0 \leq k \leq a \\ k \neq i, j}}(x-k)^{2}
$$

for some $0 \leq i<j \leq a$. Comparing the coefficients of $x^{2 a-1}$ we get

$$
\begin{aligned}
\frac{b_{a-1}}{(2 a-1) !}-\frac{b_{a}}{(2 a) !}(0 & +1+2+\ldots+2 a-1) \\
& =\frac{b_{a}}{(2 a) !}\{-(i+j)-2(0+1+\ldots+a)+2(i+j)\},
\end{aligned}
$$

which yields

$$
\frac{b_{a-1}}{b_{a}}=\frac{a^{2}-2 a+i+j}{2 a} .
$$

This ratio belongs to the interval $\left[(a-1)^{2} /(2 a),\left(a^{2}-1\right) /(2 a)\right]$, hence Lemma 4 is proved.

3. Proof of Theorem 1. We detail the proof for Stirling numbers of second kind only. For Stirling numbers of first kind, the whole argument can be repeated.

Put

$$
G(x)=(2 a) !\left\{\left(\begin{array}{c}
x \\
a+1
\end{array}\right) \widetilde{S}_{1}^{a+1}+\ldots+\left(\begin{array}{c}
x \\
2 a
\end{array}\right) \widetilde{S}_{a}^{2 a}\right\} .
$$

One can see that $G(x) \in \mathbf{Z}[x]$ and $G(x)$ is divisible by $x(x-1) \ldots(x-a)$. Let $x, y, z$ be an arbitrary but fixed solution to (1) with $x>2 b 16^{a} a^{8 a},|y| \geq 2$ and $z \geq 2$. Then

$$
G(x)=(2 a) ! b y^{z} .
$$

We have seen there are integers $0 \leq i_{1}<i_{2} \leq a$ (in the factorization of $G(x)$ over C) for which the factors $x-i_{k}, k=1,2$, are simple. Hence $G(x)$ can be expressed as

$$
G(x)=A_{1}\left(x-i_{1}\right)+\ldots+A_{2 a}\left(x-i_{1}\right)^{2 a},
$$

where $A_{1} \neq 0$. For an arbitrary $x \in \mathbf{Z}, x \neq i$, the gcd of $x-i_{1}$ and $G(x) /\left(x-i_{1}\right)$ divides $A_{1}$. We are going to give an upper bound for $\left|A_{1}\right|$.

The sum of the absolute values of the coefficients of $G(x)$ is obviously less than

$$
(2 a) !\left(\widetilde{S}_{1}^{a+1}+\ldots+\widetilde{S}_{a}^{2 a}\right) .
$$

Furthermore, by (b)

$$
\begin{aligned}
\widetilde{S}_{1}^{a+1}+\ldots+\widetilde{S}_{a}^{2 a} & <\left(\begin{array}{c}
2 a \\
a+1
\end{array}\right) \widetilde{S}_{1}^{a+1}+\ldots+\left(\begin{array}{c}
2 a \\
2 a
\end{array}\right) \widetilde{S}_{a}^{2 a} \\
& =S_{a}^{2 a} \leq s_{a}^{2 a}<(2 a) !<\left(2 a^{2}\right)^{a} .
\end{aligned}
$$


The relation $A_{1}=G^{\prime}\left(i_{1}\right)$ combined with the above inequalities implies

$$
\left|A_{1}\right| \leq 2 a\left(2 a^{2}\right)^{2 a} a^{2 a-1}<2^{3 a} a^{6 a} \quad(a \geq 1) .
$$

For a prime divisor $p$ of $x-i_{1}$ which does not divide $A_{1}$ we have $z \mid \operatorname{ord}_{p}\left(x-i_{1}\right)$. Hence we can write $x-i_{1}$ as $\left(u_{1} / v_{1}\right) y_{1}^{z}$ where $u_{1}, v_{1}, y_{1} \in \mathbf{Z}^{+}$ and $u_{1}\left|(2 a) ! b A_{1}, v_{1}\right|(2 a) ! b A_{1}$, that is,

$$
\max \left(u_{1}, v_{1}\right)<b 16^{a} a^{8 a} .
$$

Repeating the above argument we obtain

$$
x-i_{2}=\left(u_{2} / v_{2}\right) y_{2}^{z} \quad \text { with } u_{2}, v_{2}, y_{2} \in \mathbf{Z}^{+} \text {and } u_{2}\left|(2 a) ! b A_{1}, v_{2}\right|(2 a) ! b A_{1} .
$$

We may assume that $1<y_{1} \leq y_{2}$, for otherwise $x=x-i_{1}+i_{1} \leq u_{1} / v_{1}+i_{1} \leq$ $b 16^{a} a^{8 a}+a<2 b 16^{a} a^{8 a}$, which is a contradiction. Put $Y=y_{2} / y_{1}$ and $U=\left(u_{1} v_{2}\right) /\left(v_{1} u_{2}\right)$. Then we get

$$
0<\left|U Y^{-z}-1\right|=\left|\frac{\left(i_{2}-i_{1}\right) v_{2}}{u_{2} y_{2}^{z}}\right|<\frac{a v_{2}}{y_{2}^{z}} .
$$

We have two cases to distinguish: $2 y_{2} \leq Y^{2 e}$ or $2 y_{2}>Y^{2 e}$. If $2 y_{2} \leq Y^{2 e}$, then we obtain $Y>y_{2}^{1 / 2 e} \geq 2^{1 / 6}$. Since

$$
1+a \geq \frac{x-i_{1}}{x-i_{2}}=\frac{u_{2} v_{1}}{v_{2} u_{1}} Y^{z}>\frac{1}{b^{2} 16^{2 a} a^{16 a}} \cdot 2^{z / 6}
$$

the bound for $z$ is proved. In the remaining case Lemma 2 yields

(7) $\left|U Y^{-z}-1\right|>\exp \left\{-500 \cdot \log 2 y_{2} \cdot 2 e \cdot \log \left(16^{2 a} a^{16 a} b^{2}\right)(7.5+\log z)^{2}\right\}$.

Comparing (6) with (7) we obtain the bound for $z /(7.5+\log z)^{2}$.

As we have seen $S_{x-a}^{x}$ and $s_{x-a}^{x}$ have at least two simple zeros. From Lemma 4 and inequalities (e), ( $\left.\mathrm{e}^{\prime}\right)$ we deduce that the polynomials $S_{x-a}^{x}$, $s_{x-a}^{x}$ have at least three zeros with odd multiplicities, for $a>3$.

If $a=2$ then

$$
S_{x-2}^{x}=\left(\begin{array}{l}
x \\
3
\end{array}\right) \frac{3 x-5}{4}, \quad s_{x-2}^{x}=\left(\begin{array}{l}
x \\
3
\end{array}\right) \frac{3 x-1}{4}
$$

and Lemma 1 implies the remaining part of Theorem 1.

4. Proof of Theorem 2. From the relation (d) we have

$$
a^{n}-\left(\begin{array}{l}
a \\
1
\end{array}\right)(a-1)^{n}+\ldots+(-1)^{a-1}\left(\begin{array}{c}
a \\
a-1
\end{array}\right) 1^{n}=a ! b y^{z} .
$$

By taking $w_{i}=a+1-i$ we can apply Lemma 3 , and Theorem 2 is proved for $a \geq 3$. If $a=2$ our theorem is a consequence of a result of Schinzel and Tijdeman [7] since the equation $2^{n-1}-1=b y^{z}$ can be reduced to $k x^{2}-1=b y^{z}$ with $k \in\{1,2\}$. 
Acknowledgements. The authors are grateful to the referee for his valuable remarks.

\section{References}

[1] B. Brindza, On $\mathcal{S}$-integral solutions of the equation $y^{m}=f(x)$, Acta Math. Hungar. 44 (1984), 133-139.

[2] F. T. How ard, Congruences for the Stirling numbers and associated Stirling numbers, Acta Arith. 55 (1990), 29-41.

[3] M. Mignotte and M. Waldschmidt, Linear forms in two logarithms and Schneider's method, II, ibid. 53 (1989), 251-287.

[4] Á. Pintér, On some arithmetical properties of Stirling numbers, Publ. Math. Debrecen, to appear.

[5] G. Pólya und G. Szegö, Aufgaben und Lehrsätze aus der Analysis, Band I, Springer, Berlin 1925.

[6] J. Riordan, An Introduction to Combinatorial Analysis, Wiley, New York 1958.

[7] A. Schinzel and R. Tijdeman, On the equation $y^{m}=P(x)$, Acta Arith. 31 (1976), 199-204

[8] T. N. Shorey and C. L. Stewart, On the diophantine equation $a x^{2 t}+b x^{t} y+c y^{2}=d$ and pure powers in recurrence sequences, Math. Scand. 52 (1983), 24-36.

[9] - - - Pure powers in recurrence sequences and some related diophantine equations, J. Number Theory 27 (1987), 324-352.

MATHEMATICAL INSTITUTE

KOSSUTH LAJOS UNIVERSITY

H-4010 DEBRECEN, HUNGARY

Received on 9.10.1990

and in revised form on 2.1.1991

(2090) 